\title{
SISTEMA INTELIGENTE DE DESAFIOS ABERTOS - IOCS: Uma proposta de adaptação dos padrões do Método Clínico Piagetiano em Plataforma Multiagentes
}

\author{
Othon C. Bastos Filho ${ }^{1}$, Margarete Axt ${ }^{1}$, Sofiane Labidi ${ }^{3}$, \\ Paloma D. Silveira ${ }^{2}$, Andrei Rubina Thomaz ${ }^{2}$ \\ 1 Universidade Federal do Rio Grande do Sul (UFRGS) \\ Programa de Pós-Graduação em Informática na Educação (PPGIE) \\ 2Laboratório de Estudos em Linguagem, Interação e Cognição (LELIC) \\ Caixa Postal: 5071 - CEP: 90041-970 Porto Alegre - RS. \\ 3 Universidade Federal do Maranhão, Laboratório de Sistemas Inteligentes, LSI/UFMA, \\ Campus do Bacanga, 65.000-000 - São Luís-MA, Brasil. \\ othonb@hotmail.com,maaxt@ufrgs.br, labidi@uol.com.br, palomads86@yahoo.com.br. \\ andreithomaz@hotmail.com
}

\section{Resumo}

O Sistema Inteligente de Desafios Abertos (Intelligent Open Challenges System) - IOCS é uma proposta de adaptar em um sistema multiagentes alguns padrões do Método Clínico Piagetiano, em desenvolvimento pelo Laboratório de Estudos em Linguagem, Interação e Cognição LELIC/UFRGS e o Laboratório de Sistemas Inteligentes - LSI/UFMA. Tem como características funcionais simular o papel do Experimentador e ser um Objeto de Aprendizagem, ao ser re-utilizado por outras plataformas. É constituído por três partes principais: o Sistema, o Usuário e o Agente Estrategista, realizando a mediação entre o sistema e o usuário. Este Sistema tem como proposta trabalhar a aprendizagem por meio da aplicação de desafios constituídos de provas lógico-matemáticas de concepção aberta construídas de acordo com os exames de Piaget, preocupando-se em reconhecer e registrar, as razões do usuário ante a solução encontrada, além de dar visibilidade aos processos cognitivos em curso, desencadeados não apenas pelo desafio em si, mas pelo próprio diálogo, nos termos do Método Clínico, estabelecido pelos agentes com o usuário, permitindo a visualização do relacionamento entre os processos mentais cognitivos, possibilitando um suporte à docência e à formação continuada em ambientes informatizados.

Palavras-Chave: Método Clínico, Estágios Cognitivos, Desafios Abertos, Sistema Multiagente e Agente Estrategista.

\begin{abstract}
The Intelligent Open Challenges System - IOCS is an intelligent system adapted by the theoretical contexts of the Piaget Clinical Method, in development for the Laboratory of Studies in Language, Interaction and Cognition - LELIC/UFRGS and the Intelligent Systems Laboratory - LSI/UFMA. Have as characteristics to simulate the role of experimenter and be a learning object, when is reutilized for the others platforms. Building of three main parts: the System, the User and the Strategic Agent. This system has a proposal to work learning through a challenges application constituted of open conceptions logical mathematics tests constructed with agreement of Piaget exams founds in the "Reflections Abstractions" theory, has a preoccupation to recognize and to register, the user reasons in the face solution founded, beyond visible all cognitive process in sequence, in the interaction of the agents with the user, possibility a support for the teacher and the continue making in informatics ambient.
\end{abstract}

KEYWORDS: Piaget's Clinical Method, Cognitive stages, Open Challenges, Multi-agents Systems, Strategic Agent 


\section{Introdução}

De acordo com Neal (2004), as últimas tendências do E-learning apontam para as comunidades educativas o início da exploração de objetos educacionais para pensar, aprender e conhecer, buscando enfatizar as diferenças individuais a serem redescobertas. Dentro do contexto científico atual, o Método Clínico desenvolvido por Jean Piaget (1946) apresenta-se como um método contemplativo de tais tendências, ao propor analisar os mecanismos profundos do pensamento de uma criança na resolução de um determinado problema, observando o seu desenvolvimento cognitivo e a possibilidade de compreensão da lógica implicada na problemática. Com o propósito de adaptar um sistema inteligente, baseado na abordagem de Sistemas Multiagentes (SMA) introduzida pela Inteligência Artificial Distribuída (IAD), dentro do contexto teórico dos padrões do Método Clínico Piagetiano, está em construção o Intelligent Open Challenges System - IOCS (BASTOS FILHO et al., 2005b), em desenvolvimento pelo Laboratório de Estudos em Linguagem, Interação e Cognição - LELIC/UFRGS e o Laboratório de Sistemas Inteligentes - LSI/UFMA. Este Sistema tem como desafio respeitar a individualidade do processo mental cognitivo de cada usuário, registrando suas ações e reações diante das intervenções dos agentes inteligentes na resolução de provas lógicas de concepção aberta - neste trabalho, denominadas de Desafios Abertos. O protótipo do IOCS é constituído por um grupo de agentes, formando uma Sociedade de Agentes Artificiais e Humanos, trabalhando em conjunto na realização de tarefas diferentes e intervindo dentro do desafio, com o objetivo comum de mapeamento da lógica implicada na interpretação das ações de cada usuário, propiciando-os à tomada de consciência de suas ações em busca do entendimento da lógica implicada nos desafios propostos. Desta forma, há a necessidade de se alcançar um alto grau de flexibilidade e adaptação às mudanças do usuário, satisfeita com a adaptação de padrões do Método Clínico na decisão do Agente Estrategista.

\section{Pressuposto Teórico do IOCS}

O Método Clínico Piagetiano é utilizado como forma de descobrir os aspectos do funcionamento e da estruturação da mente da criança, enquanto ela organiza os objetos sobre os quais age por esta ação e atribui sentido a estes objetos, pela verbalização dos seus atos. Busca analisar os mecanismos profundos do pensamento através da verificação da estrutura de um determinado estado de desenvolvimento ou dos processos de estruturação mental na resolução de um problema, a fim de compreender o que a levou a uma determinada resposta, possibilitando a compreensão da problemática proposta. Tem como características a coleta de dados - por meio da proposição de determinadas atividades -, a observação e a propiciação de conversas com a criança, mediadas pelo experimentador, com a finalidade de seguir seu pensamento, e a análise dos dados que foram registrados sob a ótica da teoria em questão.

Piaget, ao observar os testes estandardizados, verificou que nos mesmos não importava, ao sujeito, a lógica contida no problema (ou pré-lógica). Assim, o sujeito, na tentativa de resolver o problema, até poderia estar dando a resposta correta, embora por acaso. Ou seja, responde a tal questão sem operar de modo lógico, aleatoriamente. Uma criança, ao tentar resolver determinado problema, teria respondido de "modo errado", embora seguindo uma lógica pré-operatória, centrada na percepção dos fatos - lógica transdutiva (PIAGET, 1946), em que um elemento da classe é pego para explicar o todo. A criança ainda não conseguiria estabelecer relações e coordenações entre os fatos de modo operatório, porém, ainda assim, estaria mais próxima da resposta correta do que aquele sujeito que respondeu certo ao acaso, sem ter seguido uma linha de raciocínio para chegar a tal solução.

Seguindo esse raciocínio o IOCS tem a preocupação de observar a interpretação da figuração das respostas de cada usuário para os desafios propostos, bem como, a lógica inserida nesta interpretação, configurando assim os desafios como abertos. 


\section{Sistema de Desafios Aberto Inteligente - IOCS}

O IOCS é um sistema inteligente, baseado na abordagem de Sistemas Multiagentes e adaptado dos padrões do Método Clínico Piagetiano, que utiliza provas lógicas de concepções abertas, denominadas neste trabalho de Desafios Abertos. Tem como características funcionais simular o papel do Experimentador e ser um Objeto de Aprendizagem, ao ser re-utilizado por outras plataformas.

Os Desafios Abertos são também objetos de aprendizagem, constituídos pela aplicação da lógica-matemática em um incremento lúdico (jogo), de acordo com as provas piagetianas que trabalham as Relações Lógico-Aritméticas e a Ordem das Relações Espaciais, encontradas na teoria da Abstração Reflexionante de Piaget (PIAGET, 1995).

\subsection{Principais Atores do IOCS}

É constituído por três atores principais: Sistema, Agente Estrategista e Usuário.

\subsubsection{Sistema}

Tem como função possibilitar o desencadeamento do processo cognitivo no Usuário, por meio da aplicação de Desafios Abertos, podendo ocorrer em dois momentos: quando o Sistema aplica os desafios contidos na plataforma Multiagentes ao Usuário, provocando-lhe $o$ desencadeamento do processo construtivo reflexionante, fundamentado dentro do mecanismo geral da teoria de Piaget; e quando o Sistema intervém com o Usuário, por intermédio do Agente Estrategista, levando-o a situações relativas a Tomadas de Consciência de suas ações.

A fim de alcançar o processo da abstração reflexionante pelo Usuário, o Sistema aplicará os desafios em três etapas gradativas: Observar (Para Fazer), Fazer para Compreender (Continuar) e Compreender no Pensamento (Generalizar).

$\mathrm{Na}$ etapa de Observação, o usuário, por meio de suas abstrações, fará uma observação e análise do desafio proposto, bem como dos elementos e a lógica neles implicada.

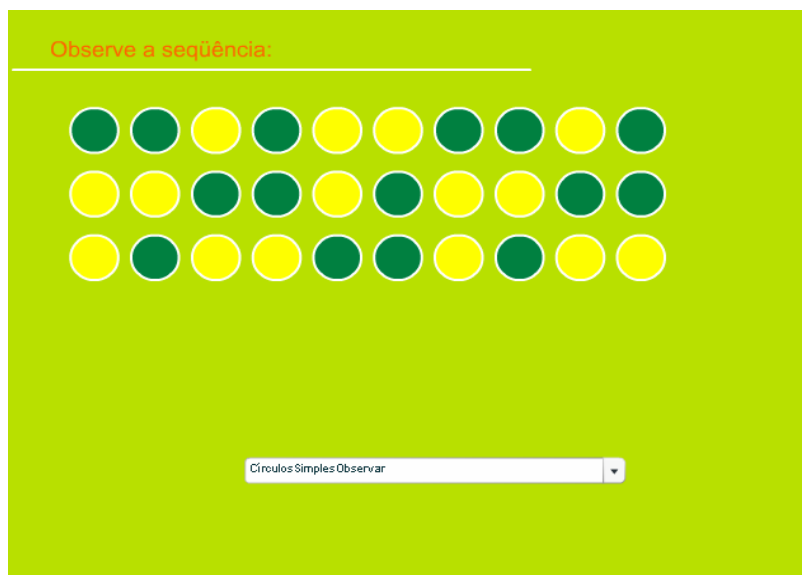

Figura 01 - Interface Observar 
Na etapa de Fazer para Compreender ou (Continuar), o Sistema irá propiciar um estímulo desafiador ao Usuário, com sequiências dando continuidade a lógica, questões baseadas na contra-argumentação, ou apresentando novos desafios, a fim de desequilibrá-lo na busca de soluções; favorecendo que o Usuário projete certas coordenações de ações hipotéticas nos desafios, sendo possível à visualização da compreensão ou somente êxito na ação.

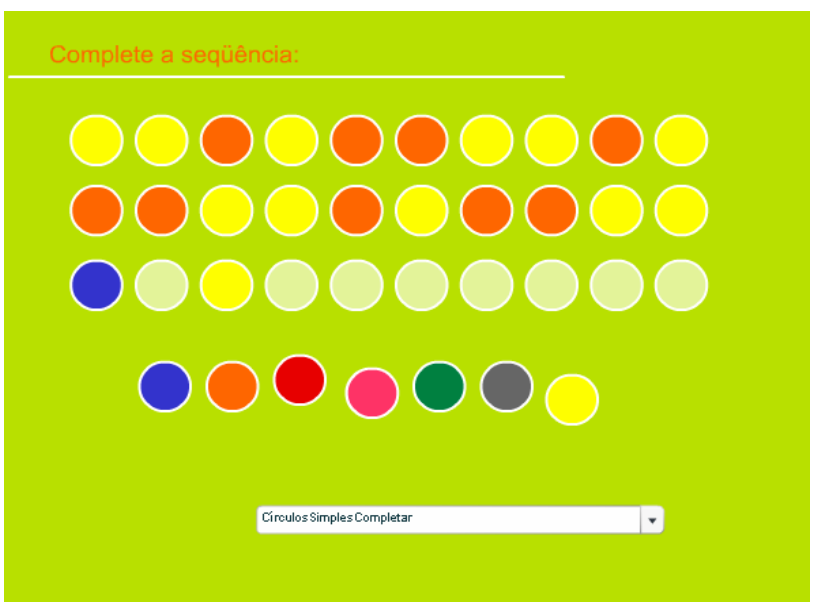

Figura 02 - Interface Fazer para Compreender

Na etapa de Compreender no Pensamento ou (Generalização), o Sistema solicita ao Usuário que generalize a lógica implicada nos desafios anteriores a fim de observar o quanto houve de compreensão da lógica, por meio da criação de novos desafios; trabalhando reflexões sobre reflexões e exigindo a Tomada de Consciência Explicitada.

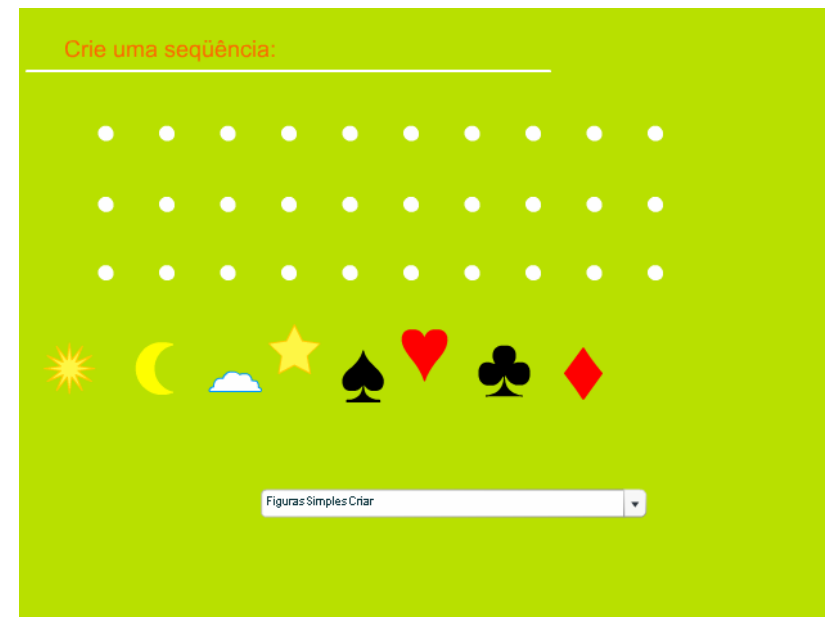

Figura 03 - Interface Compreender no Pensamento

\subsubsection{Agente Estrategista}

A arquitetura do IOCS é baseada na abordagem de Sistemas Multiagentes, introduzida pela Inteligência Artificial Distribuída, tendo seu primeiro protótipo programado na linguagem Java TM (JAVA, 2006). É formada por uma Sociedade de Agentes Humanos e Artificiais constituída pelo Usuário, Agente de Protocolo, Agente de Modelagem, Agente Estrategista e Agente de Desafios - trabalhando em conjunto na realização de tarefas diferentes e intervindo dentro do desafio, com o objetivo comum de mapeamento da lógica implicada na interpretação das ações de cada Usuário.

O Usuário, como agente humano do sistema, poderá expressar suas idéias sobre a resolução do desafio proposto e mediar a interação com outros usuários enviando suas sugestões, por meio 
de um Chat. O Agente de Protocolo é o agente responsável por registrar as ações do Usuário, obtidas pela Interface durante o processo de construção da solução do desafio. O Agente de Modelagem atua em background, analisando as ações do usuário, encontrando padrões repetitivos e categorizando-as; ao realizar a modelagem das informações, este agente cria um perfil de cada usuário para o Agente Estrategista, possibilitando com que este obtenha dados para uma posterior tomada de decisão. O Agente de Desafios gerencia as bases de dados de desafios (local de armazenamento dos desafios e seus estágios) e de questões (local de armazenamento de perguntas ou questões agrupadas em níveis de associação com as etapas dos desafios), em consonância com a estratégia selecionada pelo Agente Estrategista.

O Agente Estrategista cumpre a função de experimentador do Método Clínico dentro do IOCS, conceituando-se, neste trabalho, como um "Experimentador Virtual":

a) Mantêm ação estratégica ao reunir funcionalidades de controle para a interação do Sistema com o Usuário, por meio de suas intervenções no desafio. Avalia o perfil do Usuário, informado pelo Agente de Modelagem, com o propósito de gerar um padrão de decisão contido em umas das etapas da Interface, atuando, assim como pesquisador para o Sistema.

b) Na continuidade, informa a estratégia selecionada ao Agente de Desafios, criando um processo de problematização para o Usuário apresentando-lhe o desafio apropriado, ou ainda lhe contra-argumentando com uma nova questão, de acordo com a estratégia anteriormente selecionada, a fim de provocar-lhe desequilíbrios cognitivos, atuando como educador, dando continuidade à interação.

\subsubsection{Usuário}

O Usuário na interação com o Sistema desenvolve três funções:

a) Resolvendo os desafios - Enquanto parte do processo da Abstração Reflexionante1, o Usuário para solucionar os Desafios, terá que entendê-los, compreendendo a lógica neles implicada (BASTOS FILHO, SILVEIRA, AXT, 2004).

b) Problematizado pelo Sistema, mediado pelo Agente Estrategista, e, a partir de suas ações, é registrado um novo perfil, permitindo com que o Sistema possa interagir de modo mais adaptado ao Usuário, de acordo com o seu atual estágio cognitivo, provocando-lhe Tomadas de Consciência Conceituadas.

c) Solicitado por outro Usuário em um bate-papo para discutir e interagir com outros Usuários a respeito de melhores soluções para os desafios, e também, com novos questionamentos, com isso, age como um "agente humano" dentro do Sistema.

\section{Padrão Construtivista de Mediação (PCM)}

A interação com o Sistema pressupõe um diálogo dos agentes inteligentes com o Usuário. Dessa forma, a questão que se apresenta é de como construir categorias organizadoras das ações dos agentes, que os tornem capazes de escolher as intervenções adequadas para desencadear perturbações cognitivas nos usuários. E, ainda, como seriam essas intervenções.

Ao observar que o nível de organização das estruturas cognitivas está diretamente relacionado ao resultado alcançado pelo Usuário no Desafio, percebemos a importância de avaliar quais noções são necessárias à compreensão do mesmo, para assim desenvolver um Padrão Construtivista de Mediação (PCM), que fundamentará a ação colaborativa dos agentes, viabilizada pela característica Multiagentes do sistema IOCS.

\footnotetext{
1 Se apóia sobre as coordenações das ações do usuário, podendo estas coordenações, e o próprio processo reflexionante, permanecer inconsciente, ou dar lugar a tomadas de consciência e conceituações variadas. Fundamenta-se em dois aspectos inseparáveis: de um lado, um reflexionamento, isto é, a projeção (como um refletor) sobre objetos procedentes de ações ou operações do usuário e transferindo a um plano superior (de organização) tudo o que foi tirado de um nível inferior de atividade; e de outro lado, uma reflexão, enquanto ato mental, de reconstrução e reorganização sobre o patamar superior daquilo que é assim transferido do inferior, produzindo, desse modo, novas formas de conhecimento (PIAGET, 1995).
} 
A metodologia escolhida para desenvolver o PCM, de acordo com Bastos (2005), está calcada no Método Clínico Piagetiano, em sua variação como Método Clínico Crítico, criado e utilizado por Jean Piaget nas suas pesquisas em Epistemologia Genética. Esta metodologia tem se mostrado adequada para investigar regularidades nas condutas dos sujeitos em interação com objetos, e com base nessas regularidades, que representam maneiras de estruturação do pensamento, é possível a elaboração de categorias organizadoras das ações dos agentes. Os agentes, por sua vez, atribuirão ao Sistema, por meio do Agente Estrategistai, a capacidade de realizar o mapeamento dos processos cognitivos do usuário. Portanto, é na interação com o Sistema que se configura uma categoria específica para cada Usuário, pois o Sistema possui informações prévias sobre os possíveis modos de organização da atividade proposta, e a partir de tais informações, define um padrão de mediação de acordo com as ações realizadas pelo Usuário.

O PCM inclui todas as categorias e respectivas intervenções para um determinado jogo, situando-se na zona entre as ações do usuário e as ações do sistema. Toda a ação do usuário gera uma categoria, e toda a categoria, dentro do PCM, corresponderá a um tipo específico de intervenção, a ser utilizada pelo Sistema, adequada ao estado cognitivo do Usuário no momento de sua ação. As intervenções, por sua vez, também são elaboradas com base na metodologia clínica, a partir da análise das interações, durante a investigação clínica para construção do PCM do Desafio (intervenções do experimentador, ações e respostas verbais do Usuário). Verificamse então, quais intervenções foram mais eficientes no desencadeamento de processos de desequilíbrio e posterior Tomada de Consciência pelos Usuários. Após essa verificação, as intervenções são integradas ao Sistema em formato de mensagens, que podem ser enviadas ao Usuário por meio de uma caixa de texto.

Para essa pesquisa foi adaptada a prova "A condição da leitura de séries aditivas complexas", no entanto, foram realizadas algumas variações, que encontram suporte nas técnicas de investigação sobre a noção de ordem ${ }^{3}$. A prova consiste na apresentação de uma sequiência ordenada segundo a lei: AMAAMMAMAA...; após é solicitado ao sujeito que continue a seqüência.

Para a construção das interfaces (sete ao total), foram criadas as fases: observar (para fazer), fazer para compreender (continuar) e compreender no pensamento (generalizar). Além disso, foram utilizados três tipos de elementos para representação da ordem das seqüências, sendo estes: círculos, segmentos e figuras geométricas. Durante a realização do jogo, os usuários interagem com as três interfaces 'observar', intercaladas por interfaces 'continuar'.

Na fase 'observar', o objetivo é que o usuário inicie os processos de leitura da ordem a partir da observação. Na fase 'continuar' é enviada uma mensagem solicitando ao usuário que continue a sequiência. O objetivo é que o usuário experimente estratégias para realizar a tarefa, sendo possível visualizar as noções que ele possui a respeito da continuação da ordem. Cada ação do usuário, ou seja, seu fazer, será rastreado pelo sistema, que estará gerando um mapa de processos cognitivos ativados ${ }^{4}$.

$\mathrm{Na}$ fase 'generalizar' são apresentados diferentes elementos, e solicita-se ao usuário que organize uma seqüência, a partir do que ele conseguiu entender da ordem apresentada nas sequiências anteriores, aplicando a novos elementos, criando desafios. O objetivo é verificar qual o nível de compreensão do usuário, ou seja, verificando se ele realiza abstrações reflexionantes na leitura da ordem, sendo capaz de representação mental, aplicada posteriormente a novos conteúdos.

Depois de concluída a elaboração das interfaces, iniciam as filmagens com os Usuários.

\footnotetext{
${ }^{2}$ A prova se encontra no cap.9 do livro "Abstração Reflexionante" (PIAGET, 1995).

${ }^{3}$ A técnica se encontra no capítulo 7, do livro "A Representação do Espaço na Criança" (PIAGET, 1993).

${ }^{4}$ Foram disponibilizadas variedades de elementos para que o usuário tenha opção de escolha no momento de continuar a seqüência. Esta é uma variação da prova, visando diminuir a possibilidade do usuário continuar corretamente ou construir alternâncias simples e/ou duplas, sem que tenha realmente conseguido realizar a leitura da ordem representada, simplesmente pela falta de opções na escolha de elementos.
} 
A partir da análise das filmagens e de revisão teórica sobre os níveis de estruturação no desenvolvimento das noções espaciais relativas à ordem, bem como dos estágios de abstração reflexionante, na sua leitura, detectamos cinco estruturas de seqüências elaboradas pelos usuários. Toda essa informação que origina o PCM, geram uma tabela de ações para o Sistema e baseados nos perfis dos Usuários propiciando as ações dos Agentes no Sistema, em particular, o Agente Estrategista (SILVEIRA, P. BASTOS FILHO, O. AXT, M., 2006).

\section{Implementação do Sistema}

Para as intervenções do Agente Estrategista de forma adequada, a decisão é baseada no perfil do Usuário, categorizado de acordo com as reações dos Usuários observadas no Exame Clínico de Piaget (1926) e adaptadas, criando o PCM, adquiridos pelo Agente de Modelagem criando se assim, os perfis no Sistema, encontra-se em cinco tipos iniciais:

a) Não Importismo, quando o Usuário não provoca esforço algum de resposta;

b) Fabulação, quando o Usuário responde sem refletir, inventando uma situação não contextualizada dentro do desafio;

c) Crença Sugerida, quando o Usuário responde ao desafio sugestionado pelo Agente Estrategista, sem refletir os detalhes contidos na problematização, testemunhando, assim, apenas a sua incompreensão em relação ao mesmo;

d) Crença Desencadeada, quando o Usuário responde ao desafio por meio de seus conhecimentos anteriormente adquiridos - a resposta é um produto original de seu pensamento -, porém, necessariamente influenciado pelas questões que o Sistema propõe, forçando-o a raciocinar e a sistematizar o seu conhecimento em determinada direção, por uma situação controlada experimentalmente pelo Agente Estrategista. Enfim, o produto de raciocínio é feito sob comando, mas com recursos de materiais (conhecimentos próprios, imagens mentais, esquemas motores, pré-ligações sincréticas, entre outros) e instrumentos lógicos (estrutura do raciocínio, orientações do pensamento, hábitos intelectuais, entre outros) originários dele mesmo;

e) Crença Espontânea, quando o Usuário responde imediatamente ao desafio, não necessitando raciocinar, sendo a resposta fruto de uma reflexão anterior e original (Abstração Reflexionante), desenvolvida pelo próprio usuário em suas situações naturais de realidade de vida.

\begin{tabular}{|c|c|c|c|c|}
\hline $\begin{array}{l}\text { SIS I } \\
\text { Perfill } \\
\text { Reaçắ }\end{array}$ & $\begin{array}{l}\text { MATIZAC ÁO DAS AC } \\
\begin{array}{l}\text { Tipo de sequencia } \\
\text { /Categonias }\end{array}\end{array}$ & Feso & TERVENÇOES DOS & STEMA IOCS \\
\hline \multirow{8}{*}{$\begin{array}{c}\mathrm{E} \\
\mathrm{s} \\
\mathrm{p} \\
\circ \\
\mathrm{n} \\
\mathrm{t} \\
\hat{\mathrm{a}} \\
\mathrm{n} \\
\mathrm{e} \\
\mathrm{a}\end{array}$} & sequência certa & 0 & $\begin{array}{l}\text { Lançar próximo } \\
\text { desafio }\end{array}$ & Irtemenção \\
\hline & $\begin{array}{l}\text { sequência enada, mas } \\
\text { com as cones oometas: }\end{array}$ & & & Irtervenção \\
\hline & $\begin{array}{l}\text { a) altemâncias sinqles } \\
\text { e/ou duplas : AM, MM, } \\
\text { AM, MM }\end{array}$ & 1A & $\begin{array}{l}\text { Lançar } 1^{\circ} \text { intery. } \\
\text { (Banco } \mathrm{A})\end{array}$ & Irtemençäo \\
\hline & $\begin{array}{l}\text { b) altemâncias simples: } \\
\text { AM, AM, AM }\end{array}$ & $2 \mathrm{~A}$ & $\begin{array}{l}\text { Lançar } 1^{\circ} \text { intery. } \\
\text { (Baco } \mathrm{A})\end{array}$ & Irtemenção \\
\hline & $\begin{array}{l}\text { c) Häo apresenta } \\
\text { altemâncias s imples } \\
\text { nem duplas }\end{array}$ & $3 \mathrm{~A}$ & $\begin{array}{l}\text { Lançar } 1^{\circ} \text { interv. } \\
\text { (Banco } \mathrm{A})\end{array}$ & Irtemenção \\
\hline & $\begin{array}{l}\text { sequêtucia oom cones } \\
\text { que rä̃o es tavam na } \\
\text { sequềndia oniginal: }\end{array}$ & & & Intervenção \\
\hline & $\begin{array}{l}\text { d) utilizandosó duas } \\
\text { cones }\end{array}$ & 4A & $\begin{array}{c}\text { Lançar } 1^{*} \text { intery. } \\
(\text { Banco } A)\end{array}$ & Irtemenção \\
\hline & $\begin{array}{l}\text { e) utilizando mais de } \\
\text { duas cores }\end{array}$ & SA & $\begin{array}{l}\text { Lançay } 1^{\circ} \text { intery. } \\
(\text { Bamoo A) }\end{array}$ & Irțervenção \\
\hline
\end{tabular}

Tabela 01 - Decisão do Agente Estrategista 
A tabela acima sistematiza as ações do Agente Estrategista na busca da intervenção adequada ao desafio, de acordo com as reações do Usuário. Estas ações do Agente Estrategista são baseadas no Método Clínico Piagetiano, adaptado para a informática, mediante o registro de ações de usuários através de filmagens e análises por experimentadores ligados à pesquisa (BASTOS FILHO, 2006).

A partir da tabela são gerados os algoritmos, conforme a sequiência abaixo:

01) Se (Perfil - Reação = Espontânea) Então Faça

02) $\mathrm{Se}(\mathrm{Se} 0=$ Certa) Então Faça Peso = 0; Lançar Próximo Desafio

03) Senão

04) $\mathrm{Se}(\mathrm{Se} 0=$ Errada) Então Faça

05) Se "AE" Então Faça Peso = 1A; Lançar $1^{\circ}$ Intervenção (Banco A); Fim-se

06) Se "B" Então Faça Peso = 2A; Lançar 1 $1^{a}$ Intervenção (Banco A); Fim-se ...etc

\subsection{Arquitetura dos Agentes no JADE}

De acordo com a especificação da FIPA - (Foundation for Intelligent Physical Agents)(FIPA, 2006), um agente deve ter as seguintes características: Autonomia, reatividade, pró-atividade, social, adaptativo e cognitivo. Assim, os agentes inteligentes do IOCS implementados na plataforma JADE - (Java Agent Development Framework)(JADE,2006) responde, satisfatoriamente, aos critérios supracitados nos capítulos anteriores.

A arquitetura do IOCS é formada por quatro agentes inteligentes e de um agente humano. O Sistema Multiagentes foram desenvolvidos na plataforma JADE. O Usuário, agente humano, interage com os demais agentes artificiais (Agente de Protocolo, Agente de Modelagem, Agente Desafiador e Agente Estrategista) através de uma interface JSP. Por sua vez, os agentes inteligentes, que residem no ambiente JADE, se comunicam utilizando troca de mensagens para promover interações e intervenções adequadas para que seja alcançado o propósito do jogo. Abaixo, na figura 05 é mostrada a arquitetura do sistema multiagentes.

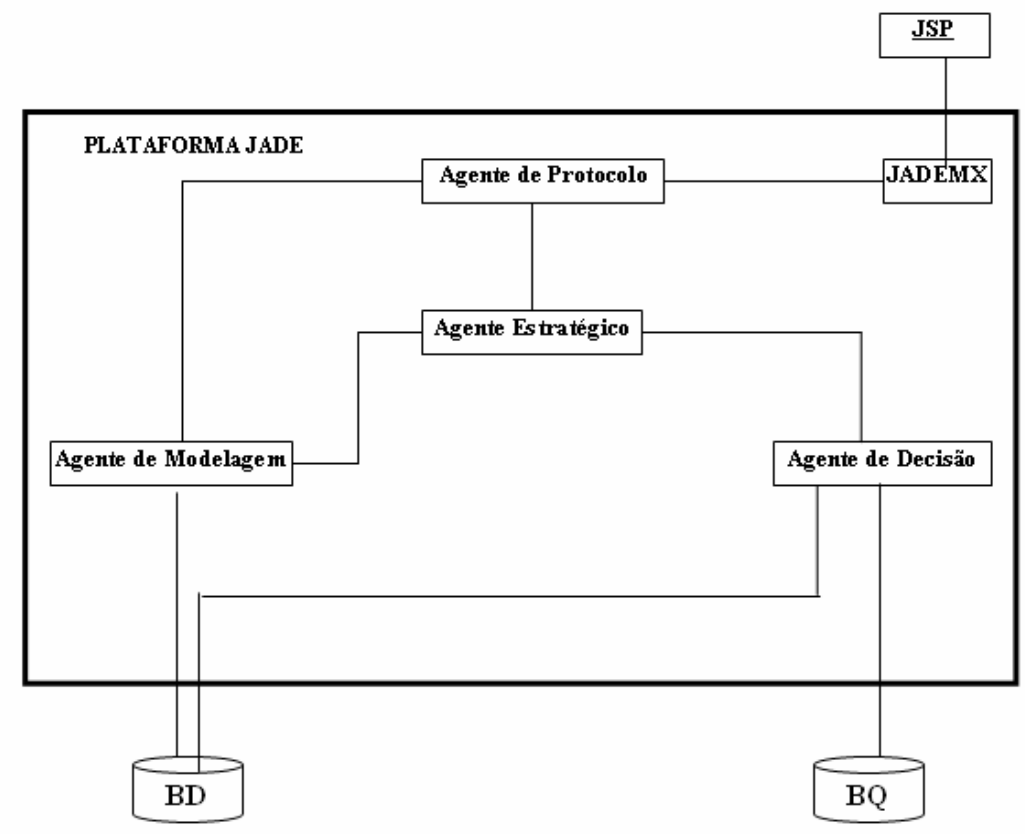

Figura 05 - Arquitetura do IOCS 
No IOCS a interação com o Usuário é realizada através da interface web desenvolvida em JSP (Java Server Page). Então, a idéia foi utilizar a tecnologia jademx para a plataforma JADE conseguir se comunicar com o meio externo, ou seja, obter as respostas do usuário na página JSP. Desta forma, os agentes inteligentes no IOCS irão interagir com o meio externo, a página JSP, em uma maneira consistente de acordo com os padrões Java.

\section{Administração do IOCS}

Na administração do IOCS, o administrador, na função Mensagem, poderá alterar as frases de intervenção de maneira a adequá-las melhor à linguagem do Usuário, a fim de obter uma melhor adaptação do Sistema para a situação na qual o Usuário se encontra.

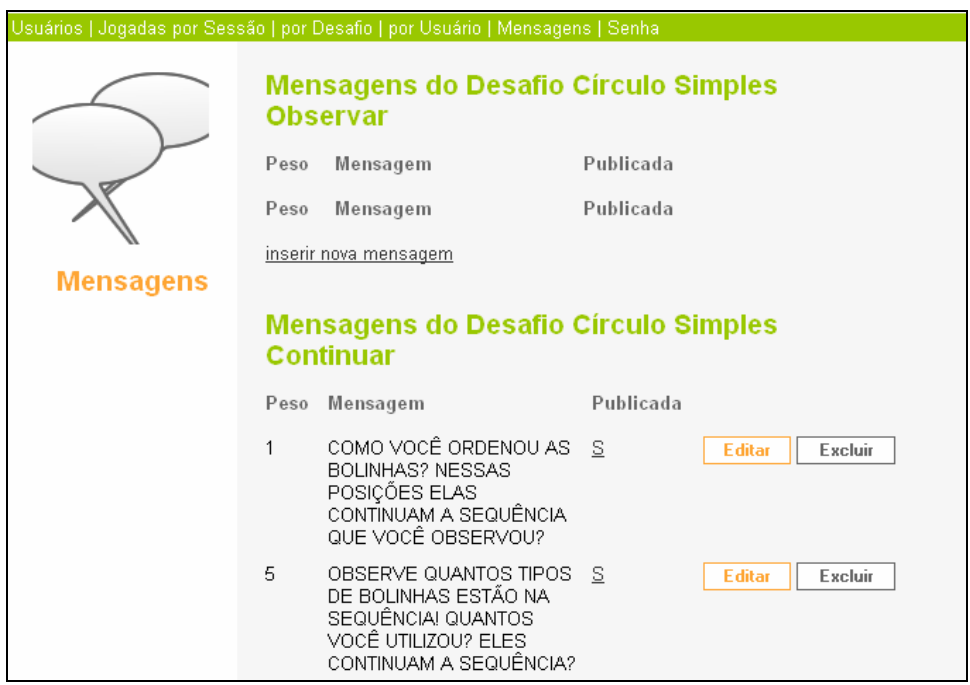

Figura 04 - Interface da função Mensagem

Ao verificar que o Usuário encontra-se em uma nova situação, o administrador, também poderá alterar os pesos da tabela com o propósito de deixar o Sistema mais sensível para aquela realidade, obtendo um melhor rendimento dos resultados. Estas ações são necessárias devido à dificuldade da aplicação do Método Clinico na informática.

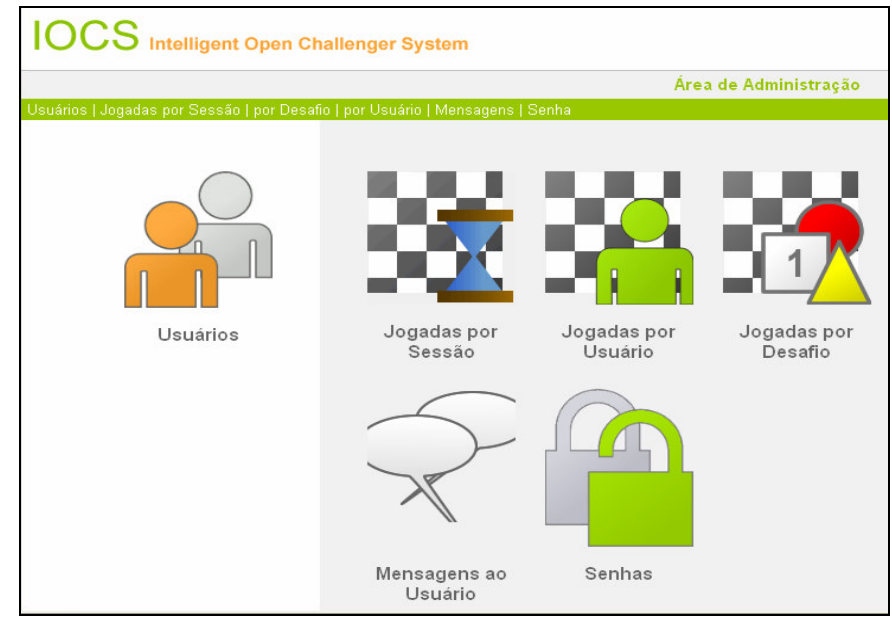

Figura 05 - Interface da Administração

Outras funções dadas ao sistema de administração: mostrar as ações do Usuário por Jogada, por sessão, por Usuário, por Desafios; além de poder mudar as senhas. Todas essas informações 
são disponibilizadas em forma de planilha, possibilitando, assim, o manejo dos dados para outros software, ou para profissionais da educação para futuras análises de interesses comuns.

A presente pesquisa encontra-se em contínuo aperfeiçoamento a fim de tornar o Sistema mais sensível a futuras possibilidades de ações dos Usuários.

\section{Conclusão}

O caráter desafiador, dentro de um fazer pedagógico, de aprender a observar e a ouvir aos aprendizes, para, então, construir, a partir do que se viu e/ou ouviu, hipóteses de trabalho que dêem consistência ao planejamento e atendam às suas necessidades e interesses, sugerem ao educador/pesquisador a necessidade da construção de um processo semelhante ao do experimentador descrito por Piaget. Baseado no exposto, o IOCS constitui-se um espaço aberto à aprendizagem, trabalhada de forma interacionista e adaptativa à construção de graus de significações por cada Usuário, através da aplicação de desafios construídos a partir de provas lógico-matemáticas, favorecendo a que se chegue a prováveis ganhos cognitivos, e, assim, tornando-se um suporte à docência e à formação continuada em Ambientes Virtuais de Aprendizagem.

\section{Referências}

AXT, M. Technology in the Education, Technology for Education: a text in construction. Computer science in the education: practical theory and, set. 2000. v. 3, n. 1, p. 51-62

BASTOS FILHO, O. C. ; AXT, Margarete ; LABIDI, Sofiane ; MACHADO, C. T. . Intelligent Open Challenges System: A possibility of adaptation of Piaget Clinical Method in Multi-agents Systems. IADIS Virtual Multi Conference on Computer Science and Information Systems, Lisboa, Pt, 2006.

BASTOS FILHO, O. C. ; Axt, Margarete ; Labidi, Sofiane ; Silveira, P. D. ; Machado, C. T. . "Systems Applied Multiagentes the Logical Challenges: A possibility of interaction through the playful increment". Magazine New Technologies in the Education, CINTED/UFRGS ISSN 1679-1916, Porto Alegre, Bra., 2005.

BASTOS FILHO, O. C.; SILVEIRA, P. D.; AXT, M. Informatizando as relações lógicoaritméticas e de ordem das relações espaciais em desafios abertos. In: IV Seminário Internacional da Região Sul - Desafios da Educação para a América Latina: Solidariedade e Educação. Pelotas, 2004.

BASTOS FILHO, O. C., Labidi, S., Fonseca, L. C., and Nascimento, E. "ICS Agents Mediated E-Commerce System". International Conference On Information Systems ICEIS. Angels, France, 2003.

FIPA. Foundation for Intelligent Physical Agents,URL: http://www.fipa.org/, último acesso em abril de 2006.

JADE. Java Agent Development Framework, URL: http://jade.cselt.it/ , último acesso em abril de 2006.

JAVA. URL: http://www.java.com. Último acesso em abril de 2006

LABIDI, S. and FERREIRA, J.S "Technology Assisted Instruction Applied to Cooperative Learning”. In the Proceedings of the IEEE International Frontiers in Education, FIE, 1998.

LABIDI, S; SILVA, J. C.; COUTINHO, L.R.; COSTA, E. B. "Agent-based tutoring system for supporting cooperative and distant learning". In Proceedings of the International Conference on Computers and Advanced Technology in Education, CATE, 2000.

10 V. 4 № 1, Julho, 2006 
PIAGET, Jean. A equilibração das estruturas cognitivas: problema central do desenvolvimento. Rio de Janeiro: Zahar, 1976.

Jean. A formação do símbolo na criança: imitação, jogo e sonho, imagem e representação. 3. ed. Rio de Janeiro: Zahar, 1978.

, Jean. A tomada de consciência. São Paulo: Melhoramentos/Edusp, 1978.

, Jean. Fazer e compreender. São Paulo: Melhoramentos, 1978.

, Jean. A representacão do espaço na crianca. Porto Alegre: Artes Médicas, 1993.

Jean. Abstração reflexionante: relações lógico-aritméticas e ordem das relações espaciais. Porto Alegre: Artes Médicas, 1995.

, Jean. A noção de tempo na criança. Rio de Janeiro: Record, 1946.

Jean. O conceito de vida. Em Piaget, J. A representação do mundo na criança. Rio de Janeiro: Record. (Original publicado em 1926) .

NEAL, L. "E-learning visionaries look to the future" Editor-In-Chief, eLearn Magazine, 2004.

SILVEIRA, Paloma D; BASTOS FILHO, Othon; AXT, Margarete. PADRÃO CONSTRUTIVISTA DE MEDIAÇÃO: Estudo do desenvolvimento de noções infralógicas espaciais topológicas durante a interação entre usuários e um sistema multiagente, com vistas à fundamentação de ações colaborativas entre eles. VI Seminário de Pesquisa em Educação da Região Sul. Universidade Federal de Santa Maria: 2006 